features with a precision of less than 100 nanometres.

Using yeast cells, the authors show that most signals from fluorescently tagged proteins are still detectable even after the cells have been embedded in resin and sectioned for electron microscopy (EM). To correlate signals from FM and EM, the researchers introduced small microspheres visible in both modalities into the samples. From these, they were able to map features found in FM to images obtained with EM.

The team used the technique to image single HIV particles on mammalian cell surfaces.

J. Cell Biol. doi:10.1083/

jcb.201009037 (2011)

\section{CANCER GENETICS}

\section{One catastrophe, many mutations}

Cancer is thought to develop through the gradual accumulation of mutations across the genome. But a small percentage of cancers may instead come about through a one-off onslaught of tens to hundreds of rearrangements to a single chromosome.

Peter Campbell at the Wellcome Trust Sanger Institute in Cambridge, UK, and his colleagues analysed the genomes of ten patients with leukaemia. One patient had several distinct patterns of genomic rearrangement, such as a concentration of mutations in one section of one chromosome, and gene copy numbers - often raised in cancer - that fluctuated between just one and two. Further analysis of more than 3,000 other patient samples revealed similar patterns in $2-3 \%$ of people with cancer.

From modelling work, the authors conclude that such patterns are consistent with a chromosome shattering into tens to hundreds of pieces, many of which are then pieced together by the cell's DNArepair machinery, leaving mistakes. Such a catastrophe could lead to many cancercausing mutations.

Cell 144, 27-40 (2011)

\section{Spinning yarns of nanotubes}

Materials such as metals and semiconductors have been spun into yarns that can be woven, sewn and tied in knots, raising the possibility of new types of textiles with electronic capabilities.

Ray Baughman and his co-workers at the University of Texas at Dallas used a low concentration of multi-walled carbon nanotubes as the 'host' material to provide durable, flexible and weavable yarns. A high concentration of 'guest' material added the desired functionality. The researchers fabricated 100-micrometrewide yarns by twist-spinning specific combinations of the two components, a method that overcomes limitations, such as lack of material durability and functionality, of previous approaches.

Using lithium iron phosphate $\left(\mathrm{LiFePO}_{4}\right)$ as the cathode (pictured) and graphene nanoribbon yarn as the anode, the authors describe how to create a weavable yarn with promising lithium-ion battery functionality.

Science 331, 51-55 (2011)

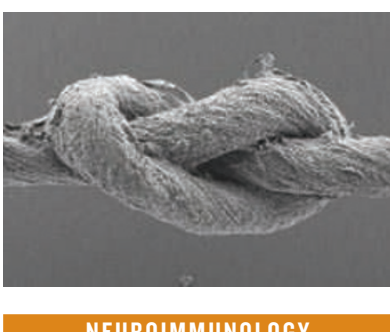

\section{Immune input} for retinal repair

Healing of the eye's retina after injury is aided by immune cells that dampen local inflammation and boost the retina's capacity for repair.

Michal Schwartz at the Weizmann Institute of Science in Rehovot, Israel, and her team monitored the influx of cells called macrophages from the blood into the damaged retinas of mice. They found that the immune cells flooded

COMMUNITY CHOICE

The most viewed papers in science

\title{
Graphene layers made to order
}

\section{HIGHLY READ \\ on apl.aip.org in November 2010}

By flinging carbon atoms at a nickel film, researchers have succeeded in generating specific numbers of layers of graphene - atomically thick sheets of carbon that

promise to surpass silicon as a material in electronic devices. Different thicknesses have markedly different properties, but controlling thickness is a challenge for current production techniques, with some able to generate only single layers.

Jene Golovchenko and his colleagues at Harvard University in Cambridge, Massachusetts, accelerated carbon atoms in an electric field and deposited doses corresponding to the amount needed for one-, two- or three-layered graphene onto nickel sheets. When heated, the carbon formed graphene of the specified thickness.

Because the underlying technology used in this method is already widespread in the fabrication of semiconductors, it could be easily used for large-scale graphene production. Appl. Phys. Lett. 97, 183103 (2010)

the retinas, with most homing in on the area of damage - the layer containing the retinal ganglion cells (RGCs). Boosting macrophage levels enhanced the survival of RGCs and triggered proliferation of retinal progenitor cells. The macrophages also altered the levels of immune regulators to decrease inflammation in the damaged retina.

The authors did not find any signs of newly generated mature retinal neurons. They therefore suggest that the progenitor cells provide a protective environment, for example by modulating the immune response, rather than replacing damaged cells.

J. Exp. Med. doi:10.1084/ jem.20101202 (2011)

\section{MOLECULAR EVOLUTION}

\section{The birth of a gene}

How new genes emerge while others decay could be explained, in part, by an unusual means of gene duplication.

Ichizo Kobayashi at the University of Tokyo and his team compared the complete genome sequences of ten strains of the bacterium Helicobacter pylori, which causes stomach ulcers, from people living around the world. From this, they reconstructed the evolutionary history of the organism's chromosome organization.

Some strains contain a single copy of a host-interaction gene, whereas others have two. The duplicate gene and the DNA between the two have been flipped around. The researchers think that a single process copied the gene while inverting the DNA. In some strains, this mechanism seems to have interrupted a hostinteraction gene, causing it to decay, whereas in other strains a new gene has evolved as a result of the process.

The same mechanism could occur in cancer cells, creating abnormal numbers and arrangements of genes, the researchers say.

Proc. Natl Acad. Sci. USA doi:10.1073/pnas.1012579108 (2011)

\section{$\rightarrow$ NATURE.COM}

For the latest research published by Naturevisit:

www.nature.com/latestresearch 